\title{
Electrodynamic Dust Shields on the International Space Station: Exposure to the space environment
}

\author{
C.I. Calle', P.J. Mackey ${ }^{1}$, M.D. Hogue', M.R. Johansen ${ }^{1}$, H. Yim², P.B. Delaune ${ }^{2}$ and J.S.
} Clements $^{3}$

1 Electrostatics and Surface Physics Laboratory, NASA, Kennedy Space Center, FL 32899

${ }^{2}$ NASA Johnson Space Center, Houston, TX 77058, USA

3 Department of Physics and Astronomy, Appalachian State University, Boone, NC 28608, USA

\begin{abstract}
Electrodynamic Dust Shields (EDS) have been in development at NASA as a dust mitigation method for lunar and Martian missions. An active dust mitigation strategy, such as that provided by the EDS, that can remove dust from surfaces, is of crucial importance to the planetary exploration program. We report on the development of a flight experiment to fully expose four EDS panels to the space environment. This flight experiment is part of the Materials International Space Station experiment X (MISSE-X), an external platform on the International Space Station that will expose materials to the space environment.
\end{abstract}

Keywords: Space applications, particle control, particle charging

\section{Introduction}

NASA's human exploration of Mars, the moon, and asteroids faces several important challenges. New technologies must be developed to protect astronauts from space radiation and to obtain commodities from local resources. Dust mitigation technologies are also high on the list of requirements for these planetary bodies, since they are covered with layers of dust that can interfere with the operation of equipment, rovers, habitats, spacesuits, and resource processing plants. In the case of Mars, the dust is lofted into its atmosphere by dust storms and dust devils. On the moon, manned and unmanned exploration activities disturb the surface dust. In both cases, it is expected that both the lofted and surface dust are electrostatically charged.

Our NASA laboratory has been developing an active dust mitigation technology called the Electrodynamic Dust Shield (EDS) since 2004 [1-5]. The EDS has been successfully tested under atmospheric conditions that simulate both Mars and the moon environments. Here, we describe the development of a flight experiment for the Materials International Space Station Experiment X (MISSE-X) to demonstrate the operation of the EDS while exposed to the space environment.

\section{The MISSE-X Mission}

The Materials International Space Station Experiment X uses an external platform to be deployed on the International Space Station (ISS) for space environmental studies designed to advance the development of materials and devices critical for space exploration (Fig. 1). MISSE-X is a follow-on to the successful MISSE series of ISS 
experiments. MISSE-X will provide plug-and-play capabilities to facilitate experiment installation, removal, and return in the post-Shuttle era [6].

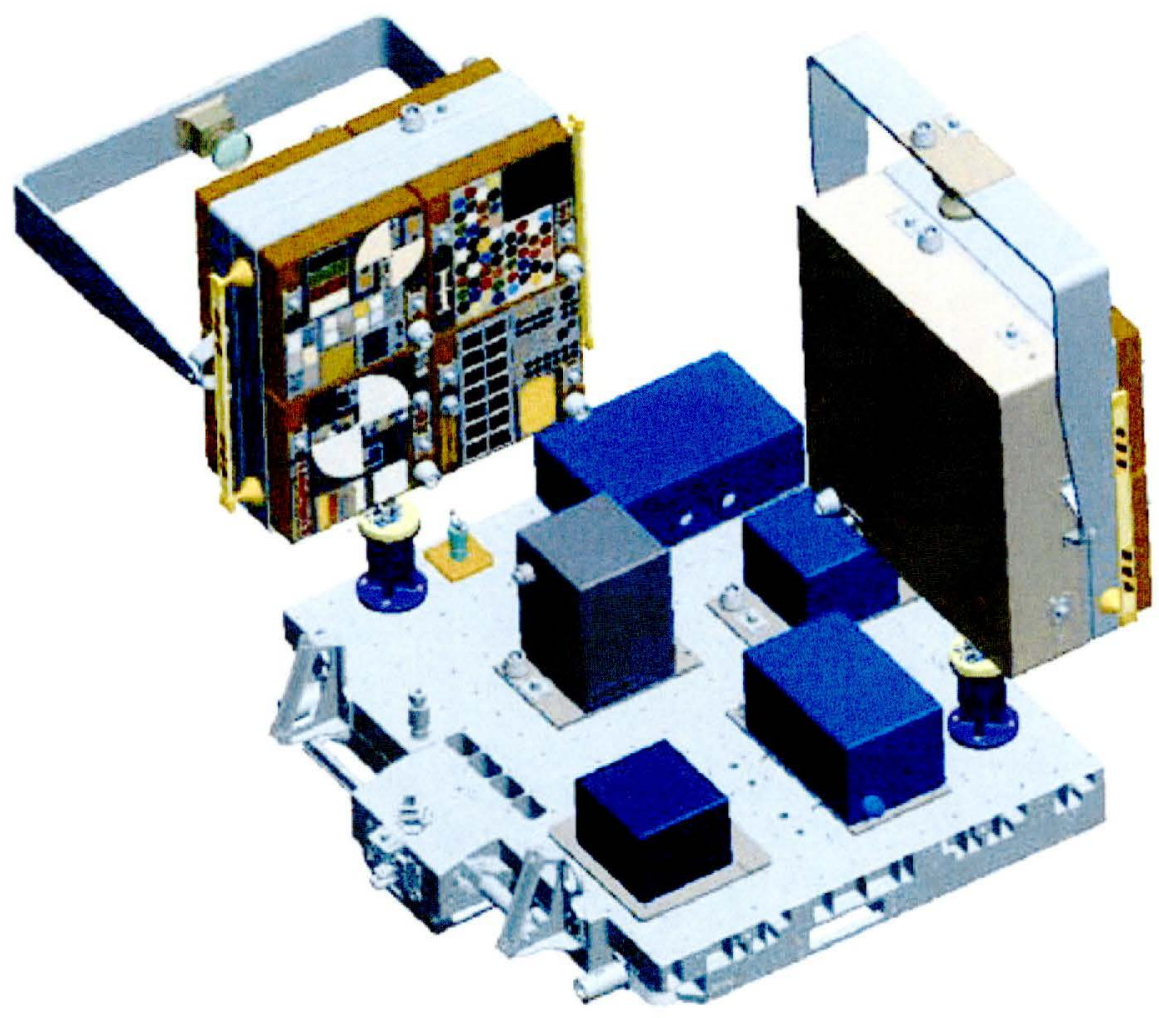

Figure 1. Preliminary design for MISSE-X [6]

MISSE-X will provide experiment exposure to the ram, wake, nadir or zenith direction. The payload will be robotically installed on ISS. Command, control, sensor and image data will be handled by the ISS communications system and ground payload operation center at NASA Marshall Spaceflight Center. Experiment modules can be replaced in orbit during subsequent flights to ISS.

\section{The Electrodynamic Dust Shield Technology}

The EDS is an active dust mitigation technology that uses traveling electric fields to transport electrostatically charged dust particles along surfaces. To generate the traveling electric fields, the EDS consists of a multilayer dielectric coating with an embedded thin electrode grid running a multiphase low frequency AC signal. Electrostatically charged particles, such as those encountered on the moon, Mars, or an asteroid, are carried along by the traveling field due to the action of Coulomb and dielectrophoretic forces. A full description of the EDS has been given elsewhere [2].

The EDS multilayer coating has been applied to metallic and electrically insulating surfaces as well as to transparent substrates (rigid and flexible). EDS with vapordeposited metallic electrodes are used for metallic surfaces such as the spacecraft surfaces coated with thermal paint and for insulating surfaces, such as flexible radiators, astronaut helmets and habitat. For optical systems, astronaut visors, and viewports, transparent, vapor-deposited indium tin oxide (ITO) electrodes are used. 
Extensive laboratory testing using simulated lunar and Martian dust has been performed in vacuum chambers evacuated to the appropriate pressures. Testing in lunar- and asteroid-like conditions is done in vacuum chambers at $10^{-7} \mathrm{kPa}$ to approximate the pressure of the rarefied lunar atmosphere or the vacuum near the surface of an asteroid Testing under Martian-like conditions is done in vacuum chambers evacuated to under 10 $\mathrm{Pa}$ and backfilled with carbon dioxide to $0.9 \mathrm{kPa}$.

Figure 2 shows the performance of transparent EDS coatings for optical systems.

Simulated lunar dust was deposited at $10^{-7} \mathrm{kPa}$ to the four panels. Activation of the EDS system clears the dust from the surface containing the spiral-shaped ITO electrodes.

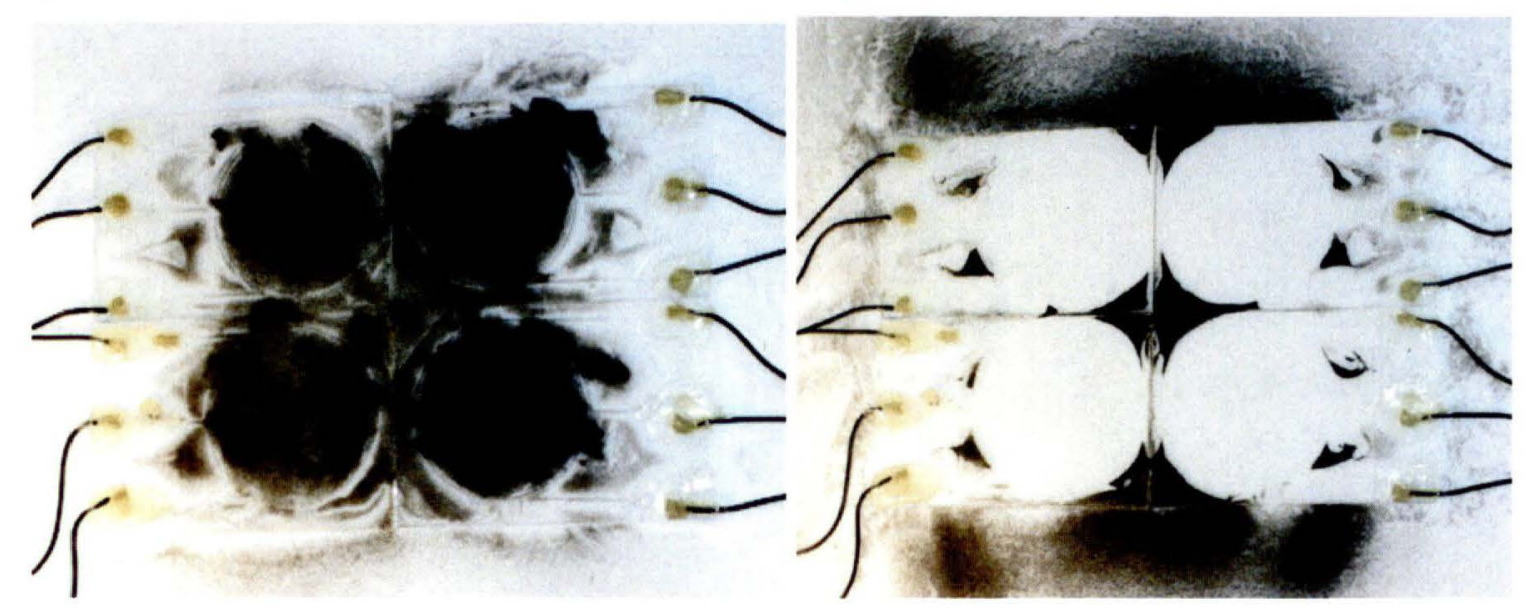

Figure 2. Testing of transparent EDS with ITO electrodes at vacuum with simulated lunar dust.

The response of a similar transparent EDS placed on a solar panel is shown in Fig. 3. Dust was deposited at $10^{-7} \mathrm{kPa}$ and the panel was activated. Obscuration and subsequent clearance of the solar panel is illustrated by the voltage output of the cell.

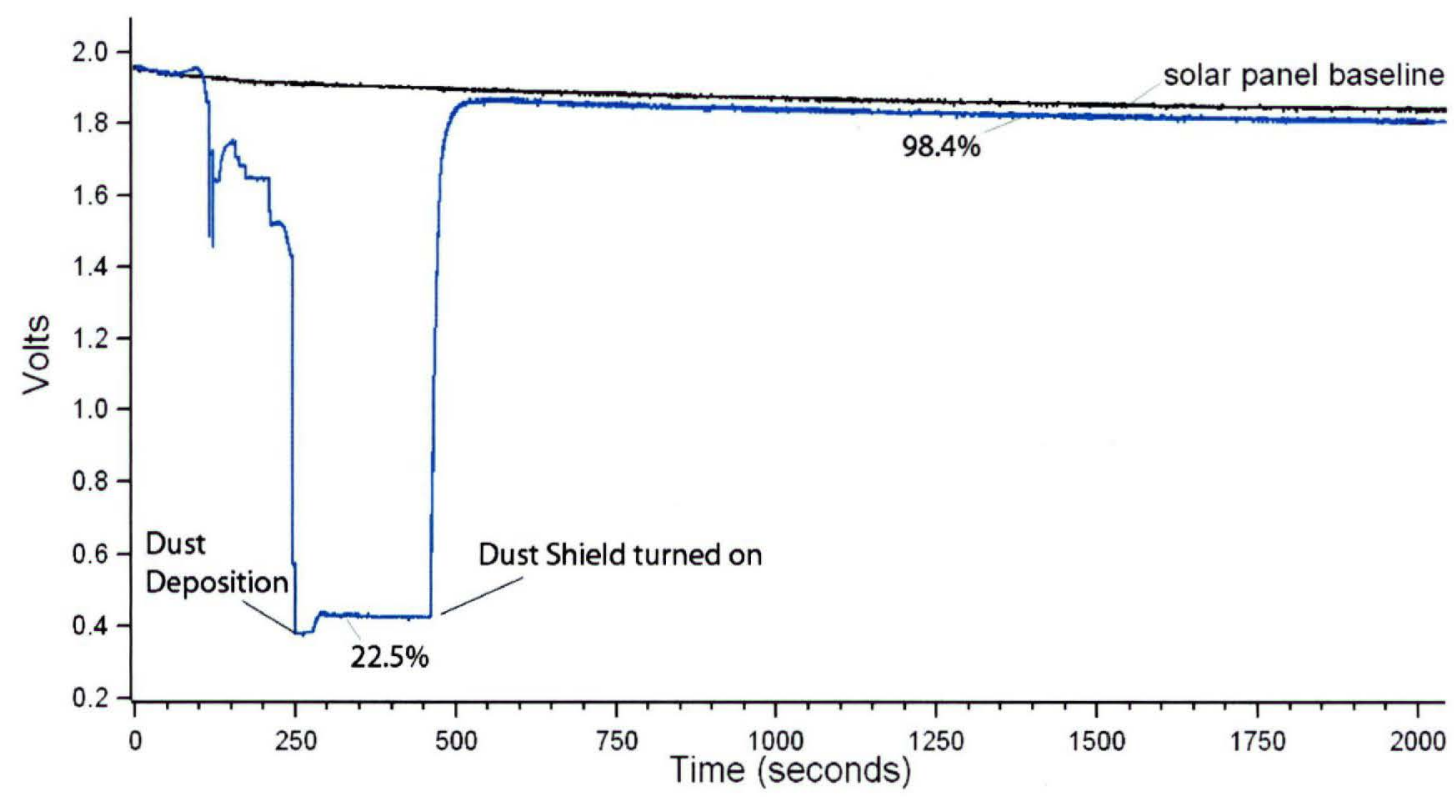


Figure 3. Solar panel response to dust loading and removal with a transparent EDS at vacuum.

EDS panels for two types of thermal radiators have also been developed and tested. These two types of radiators are rigid aluminum surfaces with thermal paint (AZ-93 or A276) or second surface mirrors consisting of aluminized fluorinated ethylene propylene (FEP). Both surfaces reflect infrared and visible light and are used to thermally insulate spacecraft and equipment. The EDS for painted radiators consists of vapor-deposited copper electrode grids on Kapton film that were attached to the aluminum surfaces prior to the application of a thermal paint. The EDS for second surface mirrors were fabricated with vapor-deposited aluminum grids on fluorinated ethylene propylene (FEP). These EDS films were attached to the aluminized FEP second surface mirror film. Figure 4 shows the reflectance measurements of an aluminum coupon coated with thermal paint and the same surface after dust deposition and removal in a vacuum chamber at $10^{-7} \mathrm{kPa}$.

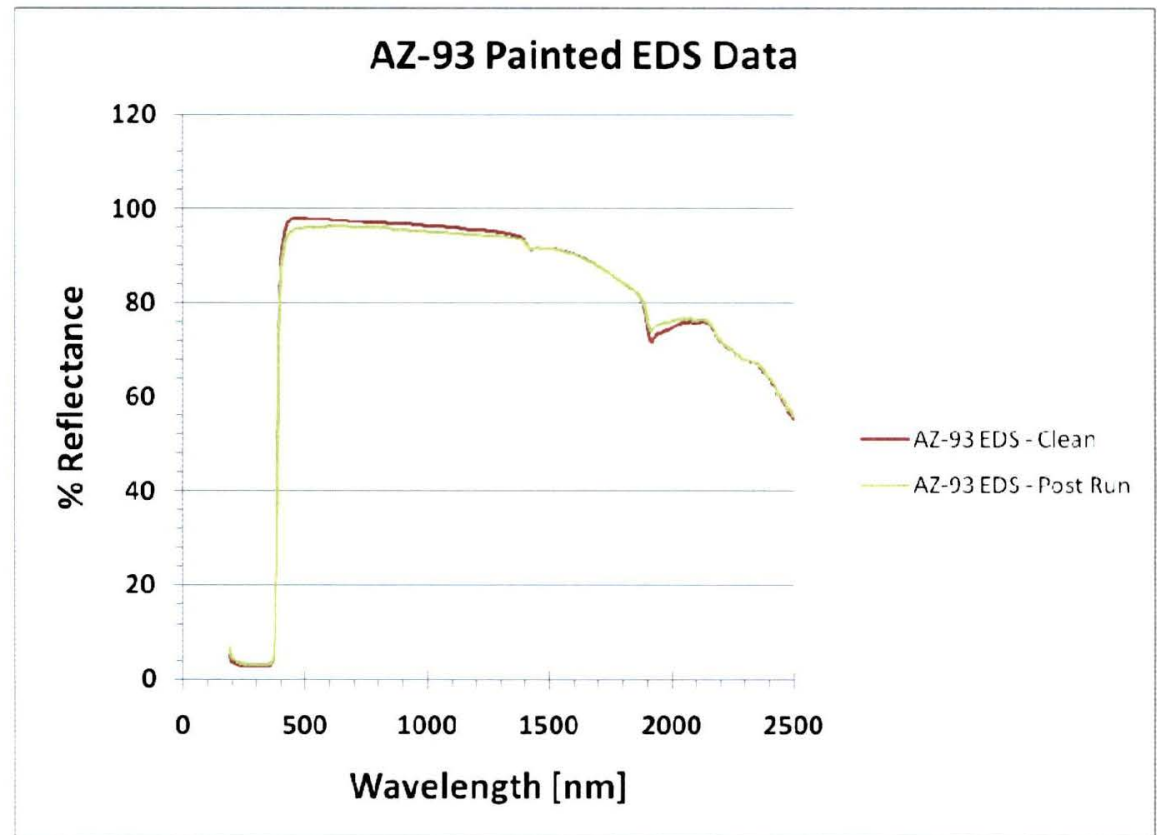

Figure 4. Reflectance spectra from $190 \mathrm{~nm}$ to $2500 \mathrm{~nm}$ for an EDS on a solid thermal radiator.

\section{The MISSE-X Payload}

Four EDS panels for four specific applications currently being developed will constitute our payload for MISSE-X: a transparent EDS for solar panels and optical systems, an EDS for painted thermal radiators, an EDS for second surface mirror films, and an EDS for spacesuits. A compact electronics package will provide the power and signal required for the operation of the EDS. This package will also contain the control and data acquisition systems for the four EDS panels. The panels will be activated daily for a few minutes. The voltage and current to each panel and the overall power consumption to the EDS power supply will be monitored during the operation of the panels. These values will be compared to baseline measurements to determine if there has been degradation in performance. These values will be recorded along with a daily visual inspection provided 
by an onboard camera. To prevent possible contamination of the other experiments, no dust will be used on the EDS payload.

The EDS experiment will be placed on the nadir direction of the MISSE-X payload as this location will experience and environment similar to that of the moon. The EDS experiment will be continuously exposed to the space environment for the duration of the mission. The EDS panels will be returned to the laboratory for post-flight inspection and testing.

\section{References}

[1] Calle C. I., C.R. Buhler, M.R. Johansen, M.D. Hogue, and S.J. Snyder, “Active dust mitigation technology for lunar and Martian exploration," Acta Astronautica 69, (2011) 1082-1088

[2] Calle C. I. C.R. Buhler, J.L. McFall, and S.J. Snyder, J. Electrostatics 67, (2009) 8992

[3] Calle C. I. A. Chen, C.D. Immer, M. Csonka, M.D. Hogue, S.J. Snyder, M.

Rodriguez, and D.V. Margiotta, "Dust removal technology demonstration for a lunar habitat," AIAA Space 2010, Anaheim, CA, 2010

[4] Calle, C.I., C.D. Immer, J. Ferreira, M.D. Hogue, A. Chen, M.W. Csonka, N. Van Suetendael, and S.J. Snyder, "Integration of the electrodynamic dust shield on a lunar habitat demonstration unit," Proceedings of the Annual Meeting of the Electrostatics Society of America, Charlotte, NC, June 2010.

[5] Calle, C.I., E.E. Arens, J.M. McFall, C.R. Buhler, S.J. Snyder, J.K. Geiger, R.A. Hafley, and K.M. Taminger, "Reduced gravity flight demonstration of the dust shield technology for optical systems," IEEE Aerospace Conference, \#1510 (2009)

[6] Thibaeault, S.A., M.P. Ashe, R.J. Saucillo, D.G. Murphy, K.K de Groth, and D.A. Jaworske, "MISSE-X: An ISS external platform for space environmental studies in the post-Shuttle era, IEEE Aerospace Conference Proceedings, 1-13 (2011) 